\title{
Path-Protection Routing and Wavelength Assignment (RWA) in WDM Mesh Networks Under Duct-Layer Constraints
}

\author{
Hui Zang, Member, IEEE, Canhui (Sam) Ou, Student Member, IEEE, and Biswanath Mukherjee, Member, IEEE
}

\begin{abstract}
This study investigates the problem of fault management in a wavelength-division multiplexing (WDM)-based optical mesh network in which failures occur due to fiber cuts. In reality, bundles of fibers often get cut at the same time due to construction or destructive natural events, such as earthquakes. Fibers laid down in the same duct have a significant probability to fail at the same time. When path protection is employed, we require the primary path and the backup path to be duct-disjoint, so that the network is survivable under single-duct failures. Moreover, if two primary paths go through any common duct, their backup paths cannot share wavelengths on common links. This study addresses the routing and wavelength-assignment problem in a network with path protection under duct-layer constraints. Off-line algorithms for static traffic is developed to combat single-duct failures. The objective is to minimize total number of wavelengths used on all the links in the network. Both integer linear programs and a heuristic algorithm are presented and their performance is compared through numerical examples.
\end{abstract}

Index Terms-Integer linear program (ILP), lightpath, optical network, protection, shared risk link group, wavelength routing, wavelength-division multiplexing (WDM).

\section{INTRODUCTION}

$\mathbf{I}$ $\mathrm{N}$ a mesh network, path-protection mechanisms usually lead to better resource utilization compared with link protection [1], [2]. The problem of dynamic lightpath establishment (DLE) is studied in [3] in a wavelength-division multiplexed (WDM) optical network with dedicated-path protection and shared-path protection. In this study, we solve the routing and wavelengthassignment (RWA) problem in a WDM mesh network under duct-layer constraints with different path-protection schemes.

There are essentially two types of fault-management techniques [1], [4], [5]: protection [1] and restoration [6]. In this paper, we refer to protection as a proactive procedure in which spare capacity is reserved during connection setup, and we refer to restoration as a reactive procedure in which the spare capacity available after the fault's occurrence is utilized for rerouting the disrupted connections. A lightpath that carries traffic during normal operation is known as a primary lightpath. When a primary lightpath fails, the connection is rerouted over

Manuscript received June 13, 2001; revised February 6, 2002; approved by IEEE TRANSACTIONS ON NETWORKING Editor G. Rouskas. This work was supported in part by the National Science Foundation under Grant ANI-98-05285.

H. Zang is with Sprint Advanced Technology Laboratories, Burlingame, CA 94010 USA (e-mail: hzang@ sprintlabs.com).

C. Ou and B. Mukherjee are with the Department of Computer Science, University of California, Davis, CA 95616 USA (e-mail: ouc@cs.ucdavis.edu; mukherje@cs.ucdavis.edu).

Digital Object Identifier 10.1109/TNET.2003.810313 a backup lightpath. Protection schemes can be classified by the type of rerouting as link-based versus path-based, or by resource sharing as dedicated versus shared. In a link-based approach, connections are rerouted around the end nodes of the failed link; in a path-based approach, a backup lightpath is selected between the end nodes of the primary lightpath. In dedicated protection, there is no wavelength sharing between backup lightpaths, while in shared protection, backup lightpaths can share wavelengths as long as their corresponding primary lightpaths are unlikely to fail at the same time.

\section{A. Duct-Layer Constraints}

In path protection, the primary path and the backup path of a connection must be fiber-disjoint so that the network is survivable under single-fiber failures. ${ }^{1}$ In practice, fibers are put into cables, which are buried into ducts ${ }^{2}$ under the ground. A fiber cut usually occurs due to a duct cut during construction or destructive natural events, such as earthquakes. When a duct is cut, normally all of the fibers in the duct fail at the same time. Hence, a network survivable to a single-fiber failure is not necessarily survivable in duct-failure scenarios. A desired backup path of a given connection should not share any duct with the primary path of the same connection.

As an example, a network with four nodes, five ducts, and six fibers is shown in Fig. 1. The link-layer topology is shown in Fig. 2. If we do not take into consideration the duct-layer topology, and we compute two fiber-disjoint paths from node 0 to node 1 purely on the link-layer topology, we might get the primary path as $0 \rightarrow 1$, and the backup path as $0 \rightarrow 2 \rightarrow 1$. However, note that link $\langle 0,1\rangle$ and link $\langle 0,2\rangle^{3}$ are going through the same duct $(0,1)^{4}$, and, hence, both may fail due to a failure on duct $(0,1)$. So, if duct $(0,1)$ fails, the connection from node 0 to node 1 loses both paths.

We must also consider the duct-layer topology when we decide whether two connections can share certain wavelengths on certain links on their backup paths. For example, there are two connections being set up in the network shown in Fig. 1, one from node 0 to node 1 , and another from node 0 to node 2 . The

\footnotetext{
${ }^{1}$ In this paper, "fiber" is considered to be bidirectional and "link" is considered to be unidirectional.

${ }^{2} \mathrm{~A}$ duct is a bidirectional physical pipe between two end nodes

${ }^{3}$ Throughout this paper, we denote by $\langle i, j\rangle$ a unidirectional link from source node $s$ to destination node $d$.

${ }^{4}$ In this example and the example in Section III, we use $(i, j)$ to denote a duct between node pair $(i, j)$ for simplicity. In the formulations in Section II, we use an identifier to identify a duct since there might be multiple ducts between the same node pair.
} 


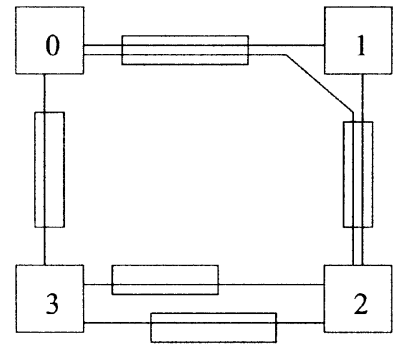

duct

fiber

Fig. 1. Example of duct-layer topology.
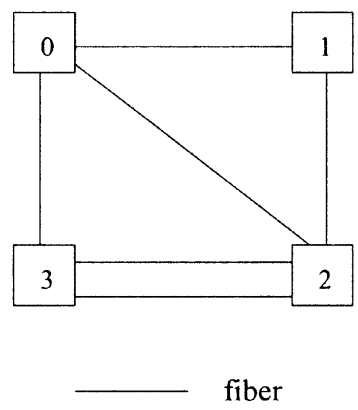

Fig. 2. Link-layer topology of the network in Fig. 1.

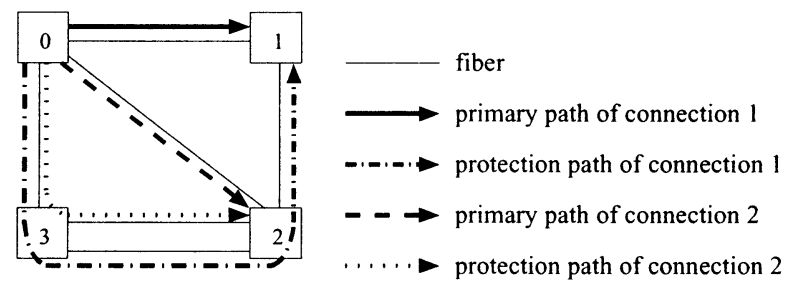

Fig. 3. Example network with two connections.

primary paths and backup paths are shown in Fig. 3. If we only look at the link-layer topology, we may allow the two backup paths to share the same wavelength on link $\langle 0,3\rangle$ because their primary paths are fiber-disjoint. However, the two primary paths actually go through the same duct $(0,2)$ and can fail at the same time. Hence, we should use different wavelengths for the two backup paths.

To summarize, in order to make a network survivable to single-duct failures, we must look at both the link-layer topology and the duct-layer topology when we compute:

- two duct-disjoint paths between the same source-destination pair;

- the backup wavelength for a connection if shared-path protection is utilized.

In general, a duct is a type of Shared Risk Link Group (SRLG) [7]-[9], which associates all the links with a failure. Multiple ducts that are buried in a Right of Way (railroad, highway, etc.) [7] might share the same failure, thus, they might belong to a SRLG. While the focus of this paper is duct-layer constraints in particular, the integer linear programs (ILPs) formulated in later sections are general enough to handle SRLG constraints (by simply viewing a duct identifier as a SRLG identifier conceptually). The heuristic proposed in Section III can also be applied to SRLG constraints provided that a graphical representation, i.e., a topology of the SRLGs, is given or can be derived.

In this paper, we solve the RWA problem [10] in a WDM mesh network under duct-layer constraints with different path-protection schemes. Although this paper considers the type of networks in which no wavelength conversion is available, i.e., under the wavelength-continuity constraint, the extension to wavelength-convertible networks is straightforward. We address the RWA problem for static traffic, and comment on the usability of some algorithms for dynamic traffic.

\section{B. Related Work}

Integer linear programming is often used to find an optimal solution when static traffic is considered [1], [11]-[15]. Several ILPs to solve the path-protection problems are developed in [1] and [11]. The programs assign wavelengths to a given set of connections according to certain protection requirements, so that the total number of wavelengths on all the links (which we refer to as total number of wavelength links) is minimized for a given network. The input to the programs includes a set of fiber-disjoint alternate routes between each source-destination pair. However, there is no description in [1] or [11] of how the alternate routes are generated. In fact, the quality of the alternate routes greatly affects how optimal the solutions of the ILPs are, as well as how fast the ILPs can be solved. The work in [12] considers two problems: determining the best backup route for each wavelength demand, given the network topology, the capacities, and the primary routes of all demands; and determining primary and backup routes for each wavelength demand to minimize network capacity and cost. ILP and distributed heuristic algorithms based on Lagrangean relaxation and subgradient optimization, under single-link or single-node failures, are presented. The work in [13] develops ILPs to minimize capacity and optimize revenue. The work in [14] considers the RWA problem with shared-link protection in a mesh network with similar objectives. Heuristics can be applied to the RWA problem with different protection schemes. In [15], the routing problem and the wavelength-assignment problem are solved separately. Several routing heuristics are developed and a vertex-coloring approach is used to solve the wavelength-assignment problem for various protection and restoration schemes in [15]. More recent work on protection routing focuses on dynamic provisioning where connection requests arrive one by one and future arrivals are not known (refer to [16]-[18] and the references therein for details).

Our work is motivated by the fact that duct-layer constraints play an important role when solving the RWA problem, as was illustrated in Section I-A. In this paper, we solve both the routing and the wavelength-assignment problems under the duct-layer constraints, using both a combined approach and a divide-andconquer approach.

\section{Organization of This Paper}

In Section II, we develop ILPs to solve the RWA problem for two path-protection schemes: dedicated-path protection and 
shared-path protection. Because the combined ILPs are too time- and-space-intensive to solve, we employ divide-and-conquer techniques to solve practical instances of the problems by partitioning the RWA problem into routing subproblem and wavelength-assignment subproblem and provide ILPs for both subproblems. In Section III, we present an efficient heuristic whose performance is comparable to the ILPs. In Section IV, we present some illustrative numerical examples. We conclude the paper and discuss a future research topic in Section V.

\section{MATHEMATICAL FormUlations}

In this section, we develop ILP formulations for the RWA problem under both dedicated-path protection and shared-path protection. We first present an ILP (ILP1) for the combined RWA problem under dedicated-path protection. We then present an ILP (ILP2) for the combined RWA problem under sharedpath protection. The objective of both ILPs is to minimize the total number of wavelength links. As the combined RWA problems are too difficult to solve for a practical-sized network, we partition the RWA problem into routing and wavelength-assignment subproblems. For the routing subproblem, we present an ILP (ILP3). For the wavelength-assignment subproblem, we present two ILPs (ILP4 and ILP5), each of which operates under different assumptions.

An ILP to solve the routing problem with the wavelengthcontinuity constraint is given in [10]. The following ILPs (ILP1 and ILP2) both contain similar routing constraints.

\section{A. Notations}

The following are given as inputs to the problem.

- $N$ : number of nodes in the network. The nodes are numbered from 1 through $N$.

- $E$ : number of links in the network.

- $D$ : number of ducts in the network.

- $W$ : number of wavelengths available on each link. The wavelengths are numbered from 1 through $W$, and the same number of wavelengths are available on all links.

- Ducts $=\{\kappa\}$ : the set of ducts in the duct-layer topology. A duct is identified by a duct identifier $\kappa$.

- Links $=\{\langle i, j\rangle\}$ : the set of links in the link-layer topology. Notation $\langle i, j\rangle$ denotes the link from source node $i$ to destination node $j$. We use $\langle i, j\rangle \in \kappa$ to denote that link $\langle i, j\rangle$ goes through duct $\kappa$. Note that, in this paper, we consider networks which can be represented by simple graphs, i.e., no two links join the same pair of nodes and no loop links. If a network has multiple links between the same pair of nodes (as in Fig. 2), we can add "pseudo nodes" onto those links and make the graph simple. Loop links are not common in communication networks, and they can be handled in the same way.

- $\Lambda=\left\{\operatorname{dem}_{i, j}\right\}$ : the traffic-demand matrix, where $\operatorname{dem}_{i, j}$ is the number of connection demands from node $i$ to node $j$. DEM $=\sum_{i, j} \operatorname{dem}_{i, j}$ represents the total number of connection demands offered to the network.

The ILPs will solve for the following variables.

- $F_{i, j}^{s, d, w}$ takes on the value 1 if wavelength $w$ on link $\langle i, j\rangle$ is carrying traffic from source node $s$ to destination $d$;
0 otherwise. These variables are employed in ILP1 and ILP2.

- $F_{i, j}^{s, d}$ denotes the number of lightpaths flowing from source $s$ to destination $d$ on link $\langle i, j\rangle$. These variables are employed in ILP3.

- $S_{i, j}^{s, d, w}$ takes on the value 1 if wavelength $w$ on link $\langle i, j\rangle$ is used to protect the connection from source node $s$ to destination $d ; 0$ otherwise. These variables are employed in ILP2.

- $f_{i, j}$ is the number of wavelengths on link $\langle i, j\rangle$ used for working traffic. These variables are employed in ILP1 and ILP2.

- $s_{i, j}$ is the number of wavelengths on link $\langle i, j\rangle$ used for backup traffic. These variables are employed in ILP2.

- $\alpha_{\kappa}^{s, d}$ denotes the number of lightpaths from source $s$ to destination $d$ on duct $\kappa$. These variables are employed in ILP1 and ILP3.

- $\lambda_{s, d}^{w}$ is the number of primary lightpaths from source node $s$ to destination node $d$ allocated on wavelength $w$. These variables are employed in ILP1 and ILP2.

- $m_{i, j}^{w}$ takes on the value 1 if wavelength $w$ is utilized by some restoration route that traverses link $\langle i, j\rangle ; 0$ otherwise. These variables are only employed in ILP2.

- $\delta_{i, j, \kappa}^{s, d, w}$ takes on the value 1 if wavelength $w$ is utilized on link $\langle i, j\rangle$ by some restoration route from node $s$ to node $d$ when duct $\kappa$ fails; 0 otherwise. These variables are only employed in ILP2.

\section{B. ILP 1: Combined ILP for Dedicated-Path Protection}

When dedicated-path protection is used, both the primary path and the backup path are carrying traffic at the same time. While we can formulate the RWA problem for dedicated-path protection similar to ILP2 in Section II-C, another way to solve the RWA problem under dedicated-path protection is to route $2 \times \operatorname{dem}_{s, d}$ lightpaths between node pair $(s, d)$ and to ensure any single-duct failure affects at most $\operatorname{dem}_{s, d}$ lightpaths between node pair $(s, d)$. Note that, in ILP1, there is no differentiation between primary traffic and backup traffic.

Objective: Minimize the total number of wavelength links:

$$
\text { Minimize } \sum_{\forall\langle i, j\rangle \in \text { Links }} f_{i, j} \text {. }
$$

\section{Subject to:}

Demand between each source-destination pair is satisfied on the primary paths

$$
2 \times \operatorname{dem}_{s, d}=\sum_{w=1}^{W} \lambda_{s, d}^{w} \quad 1 \leq s, d \leq N .
$$

Flow-conservation constraints:

$$
\begin{aligned}
\sum_{i:\langle i, j\rangle \in \text { Links }} F_{i, j}^{s, d, w} & -\sum_{k:\langle j, k\rangle \in \text { Links }} F_{j, k}^{s, d, w} \\
= & \begin{cases}-\lambda_{s, d}^{w}, & \text { if } j=s \\
\lambda_{s, d}^{w}, & \text { if } j=d \\
0, & \text { otherwise }\end{cases} \\
1 \leq w \leq W ; & 1 \leq s, d, j \leq N .
\end{aligned}
$$


A wavelength on a link can only be used by one lightpath:

$$
\sum_{1 \leq s, d \leq N} F_{i, j}^{s, d, w} \leq 1 \quad \forall\langle i, j\rangle \in \text { Links; } \quad 1 \leq w \leq W .
$$

The number of lightpaths traversing link $\langle i, j\rangle$ is

$$
f_{i, j}=\sum_{1 \leq s, d \leq N} \sum_{w=1}^{W} F_{i, j}^{s, d, w} \quad \forall\langle i, j\rangle \in \text { Links. }
$$

The number of lightpaths traversing duct $\kappa$ between sourcedestination pair $(s, d)$ is

$\alpha_{\kappa}^{s, d}=\sum_{\forall\langle i, j\rangle \in \kappa} \sum_{w=1}^{W} F_{i, j}^{s, d, w} \quad \forall \kappa \in$ Ducts; $\quad 1 \leq s, d \leq N$.

When duct $\kappa$ fails, the number of lightpaths which fail between the source-destination pair $(s, d)$ should not exceed the demand between them:

$$
\alpha_{\kappa}^{s, d} \leq \operatorname{dem}_{s, d} \quad \forall \kappa \in \text { Ducts; } \quad 1 \leq s, d \leq N .
$$

Equation (7) guarantees that at least $\operatorname{dem}_{s, d}$ can be successfully routed between node pair $(s, d)$ after one single-duct failure, which automatically implies duct-disjointness, and duct-disjointness implies link-disjointness. ${ }^{5}$

The complexity of ILP1 in terms of the number of variables ${ }^{6}$ is $O\left(N^{4} \cdot W+N^{2} \cdot D\right)$ as ILP1 has $O\left(N^{4} \cdot W\right)$ variable $F s$ and $O\left(N^{2} \cdot D\right)$ variable $\alpha s$.

Sometimes, the objective for RWA is to minimize the total number of wavelength used in the network. If that is the case, then we can substitute the objective of ILP1 with the following ( $\beta_{w}$ takes on the value 1 when wavelength $w$ is used in the network; 0 otherwise):

Minimize $\sum_{w=1}^{W} \beta_{w}$

such that $\beta_{w} \geq F_{i, j}^{s, d, w} ; 1 \leq w \leq W ; 1 \leq s, d, i, j \leq N$.

\section{ILP2: Combined ILP for Shared-Path Protection}

Objective: Minimize the total number of wavelength links:

$$
\text { Minimize } \sum_{\forall\langle i, j\rangle \in \operatorname{Links}}\left(f_{i, j}+s_{i, j}\right) .
$$

Subject to:

Demand between each source-destination pair is satisfied on primary paths:

$$
\operatorname{dem}_{s, d}=\sum_{w=1}^{W} \lambda_{s, d}^{w}, \quad 1 \leq s ; \quad d \leq N .
$$

${ }^{5}$ Two duct-disjoint paths are link-disjoint. Two duct-disjoint paths, however, do not necessarily compose two paths on the link layer. This relationship will become clear when we come to the routing heuristic in Section III-A.

${ }^{6}$ The complexity of an ILP is mainly decided by the number of variables the ILP has. The more variables there are in the ILP, the more difficult the ILP is, and the longer it takes to solve. The number of constraints does not necessarily connect to the complexity of an ILP. However, the complexity of the ILP in terms of variables should not be compared directly to the time complexity which is analyzed for the heuristic developed in Section III.
Flow-conservation constraints on primary paths:

$$
\begin{aligned}
\sum_{i:\langle i, j\rangle \in \text { Links }} F_{i, j}^{s, d, w}- & \sum_{k:\langle j, k\rangle \in \text { Links }} F_{j, k}^{s, d, w} \\
= & \left\{\begin{array}{lc}
-\lambda_{s, d}^{w}, & \text { if } j=s \\
\lambda_{s, d}^{w}, & \text { if } j=d \\
0, & \text { otherwise }
\end{array}\right. \\
1 \leq w \leq W ; & 1 \leq s, d, j \leq N .
\end{aligned}
$$

Constraints on the number of rerouted lightpaths between node pair $(s, d)$ when duct $\kappa$ fails:

$$
\begin{aligned}
& \sum_{w=1}^{W} \sum_{\forall\langle i, j\rangle \in \kappa} F_{i, j}^{s, d, w}=\sum_{w=1}^{W} \sum_{\forall e:\langle s, e\rangle \in \text { Links }} \delta_{s, e, \kappa}^{s, d, w} \\
& \forall \kappa \in \text { Ducts } \\
& \sum_{w=1}^{W} \sum_{\forall\langle i, j\rangle \in \kappa} F_{i, j}^{s, d, w}=\sum_{w=1}^{W} \sum_{\forall i:\langle i, d\rangle \in \operatorname{Links}} \delta_{i, d, \kappa}^{s, d, w} \\
& \forall \kappa \in \text { Ducts } \\
& \delta_{i, s, \kappa}^{s, d, w}=0, \\
& \forall\langle i, s\rangle \in \text { Links; } \quad \kappa \in \text { Ducts; } \quad 1 \leq w \leq W \\
& \delta_{d, e, \kappa}^{s, d, w}=0, \\
& \forall\langle d, e\rangle \in \text { Links; } \quad \kappa \in \text { Ducts; } \quad 1 \leq w \leq W \text {. }
\end{aligned}
$$

Flow conservation under wavelength-continuity constraint on backup paths:

$$
\begin{aligned}
& \sum_{\forall i:\langle i, j\rangle \in \operatorname{Links}} \delta_{i, j, \kappa}^{s, d, w}-\sum_{\forall e:\langle j, e\rangle \in \operatorname{Links}} \delta_{j, e, \kappa}^{s, d, w}=0, \\
& \quad 1 \leq j \neq s, d \leq N ; \quad \kappa \in \text { Ducts; } \quad 1 \leq w \leq W .
\end{aligned}
$$

Link $\langle i, j\rangle$ cannot protect duct $\kappa$ if it goes through duct $\kappa$ :

$$
\delta_{i, j, \kappa}^{s, d, w}=0 \quad \forall\langle i, j\rangle \in \kappa ; \quad \kappa \in \text { Ducts; } \quad 1 \leq w \leq W .
$$

Two lightpaths protected by the same wavelength $w$ on the same link $\langle i, j\rangle$ cannot go through the same duct $\kappa$ :

$$
\begin{aligned}
\sum_{1 \leq s, d \leq N} \delta_{i, j, \kappa}^{s, d, w} \leq 1, & \\
& \forall\langle i, j\rangle \in \text { Links; } \quad \kappa \in \text { Ducts; } \quad 1 \leq w \leq W .
\end{aligned}
$$

Constraints indicating whether wavelength $w$ on link $\langle i, j\rangle$ is used by some backup path:

$$
\begin{aligned}
& m_{i, j}^{w} \leq \sum_{1 \leq s, d \leq N} \sum_{\forall \kappa \in \text { Ducts }} \delta_{i, j, \kappa}^{s, d, w} \\
& N\langle i, j\rangle \in \text { Links; } 1 \leq w \leq W \\
& N \cdot N \cdot D \cdot m_{i, j}^{w} \geq \sum_{\substack{1 \leq s, d \leq N \\
\forall\langle i, j\rangle \in \operatorname{Links} ; \quad 1 \leq w \leq W .}} \delta_{i, j, \kappa}^{s, d, w},
\end{aligned}
$$


Wavelength $w$ on link $\langle i, j\rangle$ can only be utilized by either a primary path or backup paths:

$$
m_{i, j}^{w}+\sum_{1 \leq s, d \leq N} F_{i, j}^{s, d, w} \leq 1 \quad \forall\langle i, j\rangle \in \text { Links. }
$$

Constraints indicating whether wavelength $w$ on link $\langle i, j\rangle$ is used by some backup path between node pair $(s, d)$ :

$$
\begin{aligned}
S_{i, j}^{s, d, w} & \leq \sum_{\forall \kappa \in \text { Ducts }} \delta_{i, j, \kappa}^{s, d, w} \quad \forall\langle i, j\rangle \in \text { Links } \\
D \times S_{i, j}^{s, d, w} & \geq \sum_{\forall \kappa \in \text { Ducts }} \delta_{i, j, \kappa}^{s, d, w}
\end{aligned}
$$

Number of primary lightpaths traversing link $\langle i, j\rangle$ :

$$
f_{i, j}=\sum_{1 \leq s, d \leq N} \sum_{w=1}^{W} F_{i, j}^{s, d, w} \quad \forall\langle i, j\rangle \in \text { Links. }
$$

Spare capacity required on link $\langle i, j\rangle$ :

$$
s_{i, j}=\sum_{1 \leq w \leq W} m_{i, j}^{w} \quad \forall\langle i, j\rangle \in \text { Links. }
$$

The complexity of ILP2 in terms of the number of variables is $O\left(N^{4} \cdot W \cdot D\right)$ since the set of variables $\delta_{i, j, \kappa}^{s, d, w}$ dominates over other sets of variables used in ILP2.

Similar to ILP1, we can also substitute the objective of ILP2 with (8) to minimize the total number of wavelengths used in the network. For ILP2, we have the following constraint in addition to (9) because ILP2 differentiates primary paths from backup paths:

$$
\beta_{w} \geq S_{i, j}^{s, d, w}, \quad 1 \leq w \leq W ; \quad 1 \leq s, d, i, j \leq N .
$$

In solving practical-sized problems, ILP2 is too complex in terms of the number of variables and the size of the searching space. In order to make the RWA problem for shared-path protection more tractable, we divide the problem into two subproblems: the routing subproblem and the wavelengthassignment subproblem. The routing subproblem searches for two duct-disjoint paths between each source-destination pair. The wavelength-assignment subproblem assigns a wavelength to each path, and the backup paths may share wavelengths if they satisfy certain constraints. The same technique can be applied to dedicated-path protection as well.

\section{ILP3: ILP for the Routing Subproblem}

This subsection addresses the routing subproblem, and the next subsection examines the wavelength-assignment subproblem. The solution obtained from the following routing approach can be applied to both shared-path protection and dedicated-path protection. The objective here is to minimize the total capacity required for satisfying all the connection demands. While there is no concept of wavelength in ILP3, the summation $\sum_{s, d} F_{i, j}^{s, d}$ stands for the maximum number of wavelengths used on link $\langle i, j\rangle$.

Objective: Minimize the total capacity required:

$$
\text { Minimize } \sum_{\forall\langle i, j\rangle \in \operatorname{Links}} \sum_{1 \leq s, d \leq N} F_{i, j}^{s, d} .
$$

\section{Subject to:}

Flow-conservation constraints:

$\sum_{1 \leq i \leq N} F_{i, j}^{s, d}-\sum_{1 \leq k \leq N} F_{j, k}^{s, d}= \begin{cases}-2 \times \operatorname{dem}_{s, d}, & \text { if } s=j \\ 2 \times \operatorname{dem}_{s, d}, & \text { if } d=j \\ 0, & \text { otherwise }\end{cases}$ $1 \leq s, d, j \leq N$.

Any two paths between the same source-destination pair should be duct-disjoint:

$$
\alpha_{\kappa}^{s, d}=\sum_{\langle i, j\rangle:\langle i, j\rangle \in \kappa} F_{i, j}^{s, d}, \quad 1 \leq s, d \leq N ; \quad \kappa \in \text { Ducts }
$$

$\alpha_{\kappa}^{s, d} \leq \operatorname{dem}_{s, d}, \quad 1 \leq s, d \leq N ; \quad \kappa \in$ Ducts

$\operatorname{dem}_{s, d}=0,1, \quad 1 \leq s, d \leq N$.

The complexity of ILP3 in terms of variables is $O\left(N^{4}+N^{2} D\right)$ as $F_{i, j}^{s, d}$ and $\alpha_{\kappa}^{s, d}$ are the only set of variables used.

ILP3 can be varied in several ways according to different needs, as follows.

- By substituting the objective with

$$
\begin{array}{ll}
\text { Minimize } & F_{\max } \\
\text { such that } & F_{\max } \geq \sum_{s, d} F_{i, j}^{s, d}
\end{array}
$$

we can search for a load-balancing type of routing, as in [10] and [19].

- We can search for the maximal number of duct-disjoint paths between a certain source-destination pair $(i, j)$ by setting $\operatorname{dem}_{i, j}$ to 1 and leaving all the other demands as 0 . By substituting the number 2 in (29) with a different number $l$, we can decide whether or not $l$ duct-disjoint paths exist between node $i$ and node $j$. A binary search will give us the maximal value of $l$ such that $l$ duct-disjoint paths exist between node $i$ and node $j$.

- By eliminating (32) and setting the demand according to real traffic demands (in terms of number of lightpath requests), ILP3 can be used to solve the dedicated-path protection problem. This is because the total number of wavelength links in dedicated-path protection (1) is actually decided by the routing alone. Section IV compares the performance of ILP1 and ILP3.

\section{E. ILPs for the Wavelength-Assignment Subproblem}

Regarding the total number of wavelength links, the objective in dedicated-path protection (1) is determined by routing only, and any wavelength-assignment algorithm such as First-Fit [10] will do the job. If, instead, the total number of wavelengths is the objective, wavelength assignment needs to be performed carefully so that the objective can be optimized. An ILP for wavelength assignment in dedicated-path protection can be easily derived from the ILP proposed in [1] by replacing the objective with (8) and link failures with duct failures. This subsection discusses the wavelength-assignment subproblem for sharedpath protection. A good wavelength-assignment algorithm can improve sharing among backup paths and, hence, reduce the total number of wavelength links. We develop two ILP formulations to solve this problem: one for failure- dependent and the 
other for failure-independent shared-path protection. In failuredependent shared-path protection, a connection might have multiple backup paths corresponding to different failure scenarios and the primary path might share some common ducts with one or more of the backup paths. In failure-independent shared-path protection, however, a connection has two duct- and link-disjoint paths.

1) Notations: Besides $N, E, W, D$, and $\operatorname{dem}_{s, d}$, defined in Section II-A, the following additional notations are used in ILP4 and ILP5.

- $R^{s, d}$ : Set of alternate routes for node pair $(s, d)$. For a route $r \in R^{s, d}$, we use $\langle i, j\rangle \in r$ to denote that route $r$ traverses link $\langle i, j\rangle . R^{s, d}$ can be generated by ILP3 or the routing heuristic in Section III-A. For ILP5, the set of routes must be duct-disjoint. For ILP4, however, the set of routes are not necessarily duct-disjoint and can be generated by standard algorithms such as $K$-shortest path [20].

- $R_{\kappa}^{s, d}$ : Set of eligible alternate routes between node pair $(s, d)$ after duct $\kappa$ fails.

- $M$ : Maximum number of alternate routes between any node pair, i.e., $M=\max _{s, d}\left\{R^{s, d}\right\}$.

Besides $s_{i, j}$ and $m_{i, j}^{w}$, defined in Section II-A, ILP4 and ILP5 solve for the following additional variables.

- $\gamma_{w}^{s, d, r}$ takes on the value 1 if the $r^{t h}$ route between node pair $(s, d)$ is used as the primary path and is assigned wavelength $w ; 0$ otherwise. These variables are employed in ILP4 and ILP5.

- $\delta_{w, \kappa}^{s, d, r}$ takes on the value 1 if the $r^{\text {th }}$ route between node pair $(s, d)$ is used for the backup path, when duct $\kappa$ fails, and is assigned wavelength $w ; 0$ otherwise. These variables are employed only in ILP4.

- $\delta_{w}^{s, d, r}$ takes on the value 1 if the $r^{\text {th }}$ route between node pair $(s, d)$ is used for the backup path, and is assigned wavelength $w ; 0$ otherwise. These variables are employed only in ILP5.

- $w_{i, j}$ denotes the number of wavelengths used by primary lightpaths on link $\langle i, j\rangle$. These variables are employed in ILP4 and ILP5.

2) ILP4: Failure-Dependent Shared-Path Protection Formulation: This ILP formulation is derived from the ILPs in [1], by adding the duct-layer constraints.

Objective: Minimize the total capacity used:

$$
\text { Minimize } \sum_{\langle i, j\rangle \in \text { Links }}\left(w_{i, j}+s_{i, j}\right) \text {. }
$$

Subject to:

Number of wavelengths used on each link is bounded:

$$
w_{i, j}+s_{i, j} \leq W, \quad\langle i, j\rangle \in \text { Links. }
$$

Demand between each node pair is satisfied:

$$
\sum_{r \in R^{s, d}} \sum_{w=1}^{W} \gamma_{w}^{s, d, r}=\operatorname{dem}_{s, d}, \quad 1 \leq s, d \leq N .
$$

Definition of the number of primary lightpaths traversing link $\langle i, j\rangle$ :

$$
w_{i, j}=\sum_{s, d} \sum_{r \in R^{s, d}:\langle i, j\rangle \in r} \sum_{w=1}^{W} \gamma_{w}^{s, d, r}, \quad\langle i, j\rangle \in \text { Links. }
$$

Definition of the number of wavelengths used for backup paths on link $\langle i, j\rangle$ :

$$
s_{i, j}=\sum_{w=1}^{W} m_{i, j}^{w}, \quad\langle i, j\rangle \in \text { Links. }
$$

Constraints indicating whether wavelength $w$ on link $\langle i, j\rangle$ is used for some backup paths:

$$
\begin{aligned}
& m_{i, j}^{w} \leq \sum_{s, d} \sum_{\kappa \in \text { Ducts }} \sum_{r \in R_{\kappa}^{s, d}:\langle i, j\rangle \in r} \delta_{w, \kappa}^{s, d, r} \\
& \langle i, j\rangle \in \text { Links; } \quad 1 \leq w \leq W \\
& N \cdot N \cdot D \cdot M \cdot m_{i, j}^{w} \geq \sum_{s, d} \sum_{\kappa \in \text { Ducts }} \sum_{r \in R_{\kappa}^{s, d}:\langle i, j\rangle \in r} \delta_{w, \kappa}^{s, d, r} \\
& \langle i, j\rangle \in \text { Links; } \quad 1 \leq w \leq W .
\end{aligned}
$$

Only one lightpath can use wavelength $w$ on link $\langle i, j\rangle$ :

$$
\begin{aligned}
\sum_{s, d} \sum_{r \in R^{s, d}:\langle i, j\rangle \in r} \gamma_{w}^{s, d, r}+m_{i, j}^{w} & \leq 1, \\
1 & \leq w \leq W ; \quad\langle i, j\rangle \in \text { Links. }
\end{aligned}
$$

Definition of the total number of rerouted lightpaths between node pair $(s, d)$ when duct $\kappa$ fails:

$$
\sum_{r \in R_{\kappa}^{s, d}} \sum_{w=1}^{W} \delta_{w, \kappa}^{s, d, r} \geq \sum_{l \in \kappa} \sum_{\substack{r \in R^{s, d}: l \in r \\ 1 \leq s, d \leq N ;}} \sum_{w=1}^{W} \gamma_{w}^{s, d, r}
$$

Two primary paths which share the same duct cannot be backed up with the same wavelength on the same link:

$$
\begin{aligned}
& \sum_{s, d} \sum_{\substack{r \in R_{\kappa}^{s, d}:\langle i, j\rangle \in r \\
\langle i, j\rangle \in \text { Links; } ;}} \delta_{w \in \text { Ducts; } ;}^{s, d, r} \leq 1, \quad 1 \leq w \leq W .
\end{aligned}
$$

The complexity of ILP4 in terms of the number of variables is $O\left(N^{2} \cdot M \cdot W \cdot D\right)$ as the set of variables $\delta_{w, \kappa}^{s, d, r}$ has the most number of variables $\left[O\left(N^{2} \cdot M \cdot W \cdot D\right)\right]$.

3) ILP5: Failure-Independent Shared-Path Protection Formulation: Instead of failure-dependent shared-path protection, we may sometimes prefer failure-independent protection to simplify network control and management. The shared-path protection discussed in [3] is a failure-independent protection. Here, we require the primary paths sharing any common duct to take on different protection wavelengths if their backup paths go through the same link. By employing failure-independent protection, we can modify ILP4 and redefine the protection variable $\delta$ to be independent of any duct. We refer to the resulting ILP formulation as ILP5. Compared with ILP4, ILP5 has the same objective; (36)-(39) remain the same, but (40)-(44) are replaced by

$$
m_{i, j}^{w} \leq \sum_{s, d} \sum_{r \in R^{s, d}:\langle i, j\rangle \in r} \delta_{w}^{s, d, r}
$$




$$
\begin{aligned}
& N \cdot N \cdot M \cdot m_{w}^{i, j} \geq \sum_{s, d} \sum_{r \in R^{s, d}:\langle i, j\rangle \in r} \delta_{w}^{s, d, r} \\
& \langle i, j\rangle \in \operatorname{Links} ; \quad 1 \leq w \leq W \\
& \sum_{s, d} \sum_{r \in R^{s, d}:\langle i, j\rangle \in r} \gamma_{w}^{s, d, r}+m_{i, j}^{w} \leq 1, \\
& 1 \leq w \leq W ; \quad\langle i, j\rangle \in \operatorname{Links} \\
& \sum_{r \in R_{\kappa}^{s, d}} \sum_{w=1}^{W} \delta_{w}^{s, d, r} \geq \sum_{\langle i, j\rangle \in \kappa} \sum_{r \in R^{s, d}:\langle i, j\rangle \in r} \sum_{w=1}^{W} \gamma_{w}^{s, d, r} \\
& 1 \leq s, d \leq N ; \quad \kappa \in \text { Ducts } \\
& \sum_{s, d} \sum_{r \in R_{\kappa}^{s, d}:\langle i, j\rangle \in r} \delta_{w}^{s, d, r} \leq 1 \\
& \langle i, j\rangle \in \text { Links; } \quad \kappa \in \text { Ducts; } \quad 1 \leq w \leq W .
\end{aligned}
$$

The complexity of ILP5 in terms of the number of variables is $O\left(N^{2} \cdot M \cdot W\right)$ as the set of variables $\delta_{w}^{s, d, r}$ has the most number of variables $\left[O\left(N^{2} \cdot M \cdot W\right)\right]$.

When we input duct-disjoint alternate routes to ILP4 and ILP5, i.e., the backup paths will not share any duct with the primary paths between a given source-destination node pair, ILP4 and ILP5 will give the same results. However, ILP5 is much faster in finding a better solution or the optimal solution because it has a smaller search space. We illustrate this property with numerical examples in Section IV.

\section{HEURISTIC}

We develop an efficient heuristic in this section. The heuristic has three stages. In the first stage, it computes two duct-disjoint (and link-disjoint) paths for each connection demand; in the second stage, the heuristic assigns a wavelength to each path computed in the first stage; and in the final stage, the heuristic optimizes the resource utilization by iteratively rerouting some of the paths. In what follows, we will refer to the first stage as the routing heuristic and the second stage as the wavelengthassignment heuristic.

\section{A. Routing: Compute a Pair of Duct-Disjoint Paths}

Given a link-layer topology, a duct-layer topology, a source node $s$, and a destination node $d$, does there exist a pair of linkand duct-disjoint paths from node $s$ to node $d$ ? This problem turns out to be NP-complete, as shown in [17]. While the proof in [17] considers general SRG, ${ }^{7}$ it still holds if we substitute general SRG by duct. The basic idea is to reduce 3-SAT [21] to this problem by considering a clause as a duct and a literal as a link.

Since the existence of the problem is NP-complete, we develop a heuristic of reasonable performance to compute a pair of linkand duct-disjoint paths. We call the performance "reasonable" for two reasons: 1) because of the NP-completeness of the problem, the heuristic is not guaranteed to find a solution and 2) the solution found by the heuristic is not guaranteed to be optimal. The basic idea of the algorithm is to compute a pair of duct-disjoint paths and map the paths to the link layer. To compute a pair of ductdisjoint paths, the heuristic uses Suurballe's algorithm [22]. The problem statement of Suurballe's algorithm follows.

${ }^{7}$ General $S R G$ is defined as "a concept that expresses the risk relationship associating all the optical channels with a single failure" in [17].
Given a graph $G=(V, E)$ where $V$ is the vertex set and $E$ is the edge set, find a pair of edge-disjoint paths from vertex $s$ to vertex $d$ such that the total edge cost of the two paths is minimal among all such path pairs.

Suurballe's algorithm runs in $O\left(N^{2} \log N\right)$ time, where $N$ is the number of vertices in graph $G$.

We adopt Suurballe's algorithm to compute duct-disjoint paths. The inputs to the heuristic are a duct-layer topology graph, a link-layer topology graph, a source node $s$, and a destination node $d$. The heuristic either computes a pair of duct- and link-disjoint paths from node $s$ to node $d$, or returns failure. The heuristic consists of three steps, as follows.

Step 1) On the duct-layer topology, compute a pair of edgedisjoint paths from vertex $s$ to vertex $d$, using Suurballe's algorithm.

Step 2) Each link is a concatenation of ducts, and each path we have for now is also a concatenation of ducts. If we consider each duct as a character and both concatenations as strings, we may find that some links appear as a sub-string in a given path. We can now use any standard string-matching algorithm (such as KMP [23]) to replace the substring of the paths with the corresponding links, until no such replacement is possible.

Step 3) Now check the paths to see if every adjacent pair of vertices are connected by a link in the link-layer topology. If so, output the two paths and return true; otherwise, return false.

Note that the heuristic is not guaranteed to be successful for two reasons.

1) There might not exist two duct-disjoint paths between a node pair.

2) Even if there does exist a pair of duct-disjoint paths, they might not have the correct mapping to the link layer, because some ducts or concatenation of ducts do not map to links or concatenation of links. For example, in Fig. 4, a pair of duct-disjoint path from node 0 to node 1 would be $0 \rightarrow 1$ and $0 \rightarrow 2 \rightarrow 1$. However, the duct path $0 \rightarrow 2 \rightarrow 1$ does not have a mapping to the link layer.

There are two sufficient (but not necessary) conditions for the heuristic to be successful.

1) There exists a pair of duct-disjoint paths between a node pair, and

2) For each edge $(i, j)$ in the duct-layer topology, there is a corresponding edge $(i, j)$ (which corresponds to link $\langle i, j\rangle$ and link $\langle j, i\rangle)$ in the link-layer topology. For example, in Fig. 4, if there exists link edges $(0,2)$ and $(2,1)$, then the heuristic will be successful.

Note that, due to the NP-completeness of the problem, no polynomial-time algorithm can guarantee to find a pair of linkand duct-disjoint paths even if such paths exist in the network, assuming $P \neq N P$.

If we use the KMP algorithm for string matching, the computational complexity of this routing heuristic is $O\left(N \cdot E \cdot D+N^{2} \log N\right)$. Specifically, the complexity of Step 1 is $O\left(N^{2} \log N\right)$; the complexity of Step 2 and Step 3 combined is $O(N \cdot E \cdot D)$. (We can implement Step 2 and Step 3 using a shortest-path-computation-like algorithm.) 


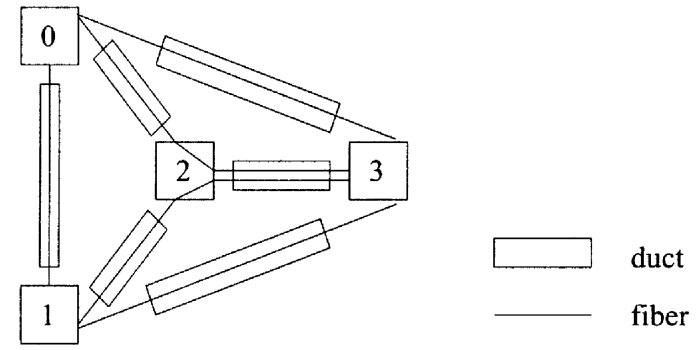

Fig. 4. Example of a pair of duct-disjoint paths which cannot be mapped to a pair of link-disjoint paths.

\section{B. Wavelength Assignment}

This subsection provides a heuristic algorithm for assigning wavelengths to connections with the input of a pair of duct-disjoint paths between each source-destination pair. The objective is still to minimize the total number of wavelength links. In order to minimize the total resource usage, the heuristic tries to minimize the resources used for primary connections, as well as to maximize the sharing among the backup resources. This wavelength-assignment heuristic works as follows.

Step 1) Remove a random connection $c$ from the connection list.

Step 2) Use First-Fit to assign a wavelength to the primary path of $c$.

Step 3) Use Last-Fit to assign a wavelength to the backup path of $c$.

Step 4) If the connection list is not empty, go to Step 1; otherwise stop.

Note that the First-Fit and Last-Fit algorithms work differently for a primary or a backup connection. Reference [3] illustrates these procedures in detail. There are several variations (e.g., sort all the connections according to the total length of their two paths in descending order before Step 1) to the above heuristic. We tried some of them, and they all have similar performance. We expect this wavelength-assignment heuristic to be representative.

Since the computational complexity of both First-Fit and Last-Fit (for a path) is $O(W \cdot N)$, the computational complexity of this wavelength-assignment heuristic is $O(Q \cdot W \cdot N)$, where $Q$ is the number of connection demands and $W$ is the number of wavelengths on each fiber.

\section{Optimization}

After wavelength assignment, the heuristic applies an iterative optimization procedure to reduce the total number of wavelength links by rearranging, if necessary, the primary and backup lightpaths. A similar approach can be found in [12]. In Phase I of this two-phase procedure, we reroute the backup lightpaths to maximize backup sharing. In Phase II, we rearrange the primary lightpaths since there may be a shorter path available after rerouting the backup lightpaths.

Phase I starts with randomly picking one connection, say, $k$, with primary lightpath $p_{p}^{k}$ and backup lightpath $p_{b}^{k}$. Next, remove the backup lightpath $p_{b}^{k}$ and update the cost of wavelength $w$ on link $\langle i, j\rangle, C_{i j}^{w}$, as follows $(+\infty$ is a large number, e.g., the diameter of the network times the maximum link cost, where the diameter of the network is defined as the total number of hops of the longest path in the network; $C_{i j}$ is the original cost of link $\langle i, j\rangle$.$) :$

$\begin{aligned} C_{i j}^{w}= & \left\{\begin{array}{l}+\infty, \\ \\ C_{i j}, \\ 0,\end{array}\right.\end{aligned}$

if $p_{p}^{k}$ traverses link $\langle i, j\rangle$, or wavelength $w$ on link $\langle i, j\rangle$ is used by a primary lightpath, or wavelength $w$ on link $\langle i, j\rangle$ is used by some backup lightpath whose primary lightpath is in the same duct as $p_{p}^{k}$ if wavelength $w$ on link $\langle i, j\rangle$ is not used otherwise.

Then, compute the minimal-cost path from the source to the destination on each wavelength layer, and pick the one with the minimal cost as the backup lightpath. Note that the link- and duct-disjoint constraints are ensured by the cost function, and the newly computed backup has a cost no larger than $p_{b}^{k}$. Finally, repeat this process for a predefined number of times or until it converges, i.e., the new backup lightpath does not have smaller cost compared with the previous one for a predefined number of times.

Phase II is similar to Phase I, except that we remove the primary lightpath $p_{p}^{k}$ and update the cost of wavelength $w$ on link $\langle i, j\rangle$ according to the following cost function:

$C_{i j}^{w}=$

$$
\begin{cases}+\infty, & \text { if } p_{b}^{k} \text { traverses link }\langle i, j\rangle, \text { or } p_{b}^{k} \text { shares some } \\ & \text { wavelength link with some backup lightpath } \\ & \text { whose primary lightpath traverses link }\langle i, j\rangle, \\ & \text { or wavelength } w \text { on link }\langle i, j\rangle \text { is used by } \\ & \text { some lightpath } \\ C_{i j}, & \text { otherwise. }\end{cases}
$$

The computational complexity of this stage largely depends on the paths generated by the routing heuristic. From our experiments, however, we observe that: 1) The gain from Phase I after repeating the process for $5 \cdot Q$ times is marginal, where $Q$ is the number of connection demands and 2) the gain from Phase II is marginal. Observation 1 implies that Phase I converges very fast. The reason observation 2 is true is that the heuristic chooses the shorter one of the two paths as primary path. Assuming Phase I repeats the process for $O(Q)$ times, the computational complexity of this optimization procedure is $O\left(Q \cdot W \cdot\left(N^{2}+N \cdot Q^{2}\right)\right)$. Specifically, the complexity of calculating the cost function for each wavelength link on one wavelength layer is $O\left(N \cdot Q^{2}\right)$. The complexity of the shortest-path algorithm is $O\left(N^{2}\right)$. Since we need to repeat the shortest-path computation (including calculating the cost function) on each wavelength layer for $O(Q)$ times, the complexity of this optimization procedure is $O\left(Q \cdot W \cdot\left(N^{2}+N \cdot Q^{2}\right)\right)$.

To conclude, the computational complexity of the heuristic is $O\left(Q \cdot N \cdot\left(N \log N+E \cdot D+W \cdot N+W \cdot Q^{2}\right)\right)$.

\section{ILlUSTRATIVE NUMERICAL EXAMPLES AND DISCUSSION}

In this section, we present numerical examples to illustrate the performance of the ILP formulations and the heuristic described in the previous sections. We first apply the ILP formulations and the heuristic on the three networks shown in Fig. 5 and compare their performance. We then apply the heuristic to three larger networks and examine its results. 


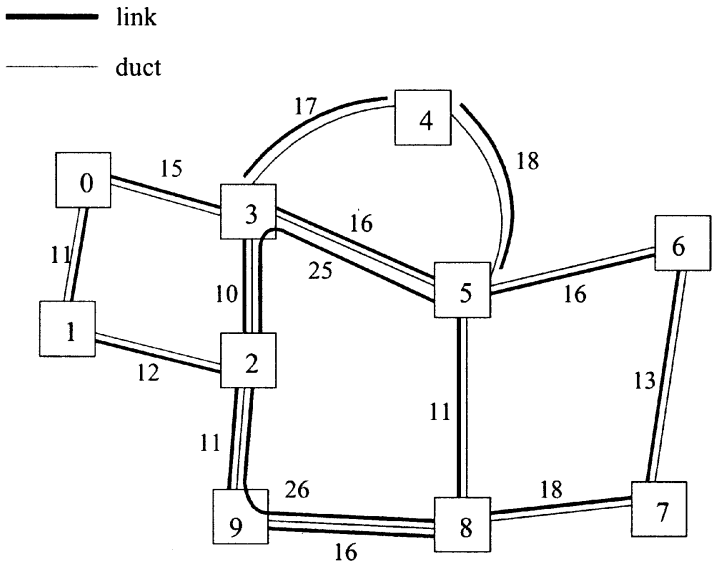

(a)

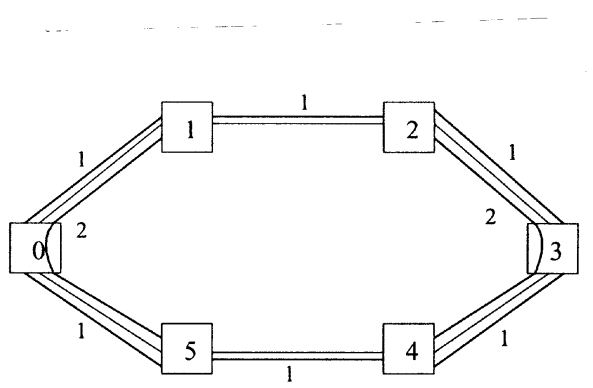

(b)

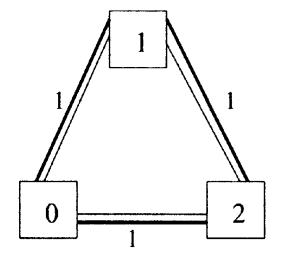

(c)

Fig. 5. Three example networks. (a) Network 1: a 10-node network. (b) Network 2: a 6-node network. (c) Network 3: a 3-node network.

TABLE I

RESULTS FROM ILP1 (COMBINED ILP FOR DEDICATED-PATH PROTECTION), ILP3 (ROUTING ILP), AND ROUTING HEURISTIC (W: NUMBER OF WAVELENGTHS, DEM: NUMBER OF CONNECTION DEMANDS)

\begin{tabular}{l|c|c|c|c|c}
\hline \hline & W & DEM & ILP1 & ILP3 & $\begin{array}{c}\text { Routing } \\
\text { Heuristic }\end{array}$ \\
\hline Network 1 & 20 & 45 & 229 & 229 & 232 \\
\cline { 2 - 6 } & 10 & 20 & 99 & 99 & 100 \\
\hline Network 2 & 15 & 15 & 70 & 70 & 70 \\
\hline Network 3 & 3 & 3 & 9 & 9 & 9 \\
\hline \hline
\end{tabular}

We use CPLEX [24] to solve the above ILPs. Tables I-III show the results reported by CPLEX when solving the various ILP formulations. The CPLEX solver was run on a computer with a $1.7-\mathrm{GHz}$ Pentium III processor and 2-GB RAM. CPLEX stops when it finds the optimal solution. If it cannot find the optimal solution within some time period or available RAM space, it stops and reports the best solution it has found so far. In the tables, numbers that are asterisked indicate the current best solution reported by CPLEX in three hours, and numbers without asterisks indicate the optimal solution found.

Table I shows the results from ILP1, ILP3, and the routing heuristic. (In the table, DEM denotes the number of connection demands chosen at random between the various node pairs in the networks.) First, we find that ILP3 gives exactly the same results as ILP1. This verifies that the total number of wavelength links in dedicated-path protection is only determined by the routing results, not wavelength assignment. Second, we observe from Table I that the performance of the routing heuristic is very close to that of ILP3 in these example networks. In practice, we can use the routing heuristic to solve the dedicated-path-protection problem as well as the routing problem when shared-path protection is used. In the rest of the paper, we use the routing heuristic to generate the input of alternate routes to ILP4 and ILP5.
TABLE II

Results From ILP2 (COMBINED ILP FOR ShARED-PATH PROTECTION) AND HEURISTIC

\begin{tabular}{l|c|c|c|c}
\hline \hline & W & DEM & ILP2 & Heuristic \\
\hline Network 1 & 2 & 3 & 13 & 14 \\
\hline Network 2 & 4 & 6 & 23 & 24 \\
\hline Network 3 & 2 & 3 & 8 & 8 \\
\hline \hline
\end{tabular}

TABLE III

RESUlts From ILP4 (FAILURE-DEPENDENT SHARED-PATH PROTECTION ILP), ILP5 (FAILURE-INDEPENDENT SHARED-PATH PROTECTION ILP), AND HEURISTIC WITH TWo DUCT-DisJoINT ALTERNATE ROUTES

\begin{tabular}{l|c|c|c|c|c}
\hline \hline & W & DEM & ILP4 & ILP5 & Heuristic \\
\hline Network 1 & 20 & 45 & $218^{*}$ & $200^{*}$ & 201 \\
\cline { 2 - 6 } & 10 & 20 & $91^{*}$ & 86 & 86 \\
\hline Network 2 & 10 & 15 & $63^{*}$ & 58 & 58 \\
\hline Network 3 & 4 & 3 & 8 & 8 & 8 \\
\hline \hline
\end{tabular}

Table II shows the results from ILP2. We observe that there are too many constraints and variables, even for a ten-node network (Network 1) with only three connection demands. Our results (not shown here) indicate that ILP2 is time and space intensive. As a result, when solving the RWA problem with shared-path protection for practical-sized networks, we have to use heuristic approaches and/or divide-and-conquer techniques to make the problem more tractable. The solutions obtained by the heuristic are also recorded in Table II.

Table III compares the results from ILP4, ILP5, and the heuristic. We observed from our experiments that ILP5 can be solved faster than ILP4 if the input of alternate paths are duct-disjoint. However, if the given alternate paths are not duct-disjoint, ILP5 will not be able to find a solution while ILP4 might. This is because, in ILP4, the backup path is solved for each failed duct. Even if there is no common backup path 
TABLE IV

Results From the Heuristic ON THREE LARGER NETWORKS. (TOTAL: TOTAL NUMBER OF WAVELENGTH-LINKS; PRIMARY: NUMBER OF WAVELENGTH-LiNKS USED FOR PRIMARY PATHS; BACKUP: NUMBER OF WAVELENGTH-LINKS USED FOR BACKUP PATHS)

\begin{tabular}{|c|c|c|c|c|c|c|c|c|}
\hline & $\bar{N}$ & $\bar{E}$ & $\overline{\mathrm{W}}$ & $\overline{\mathrm{D}}$ & $\overline{\overline{D E M}}$ & TOTAL & PRIMARY & BACKUP \\
\hline \multirow[t]{3}{*}{ Network 4} & \multirow[t]{3}{*}{19} & \multirow[t]{3}{*}{62} & \multirow[t]{3}{*}{32} & 31 & 100 & 481 & 262 & 219 \\
\hline & & & & 29 & 100 & 492 & 266 & 226 \\
\hline & & & & 27 & 100 & 522 & 266 & 256 \\
\hline \multirow[t]{4}{*}{ Network 5} & \multirow[t]{4}{*}{24} & \multirow[t]{4}{*}{86} & \multirow[t]{4}{*}{32} & 43 & 100 & 503 & 297 & 206 \\
\hline & & & & 41 & 100 & 504 & 298 & 206 \\
\hline & & & & 40 & 100 & 516 & 299 & 217 \\
\hline & & & & 39 & 100 & 532 & 303 & 229 \\
\hline \multirow[t]{2}{*}{ Network 6} & \multirow[t]{2}{*}{31} & \multirow[t]{2}{*}{94} & \multirow[t]{2}{*}{32} & 47 & 150 & 1076 & 561 & 515 \\
\hline & & & & 45 & 150 & 1103 & 561 & 542 \\
\hline
\end{tabular}

for all failure scenarios, there might be enough protection resources to work under different failure scenarios. The difference between ILP4 and ILP5 is essentially the tradeoff between failure-dependent protection and failure-independent protection: the former trades complexity for resource efficiency while the later trades resource efficiency for simplicity [25]. Table III also shows that the heuristic performs very close to ILP4 and ILP5 in the example networks.

We also apply the heuristic to three larger networks and study the effect of adding duct-layer constraints to routing. For each network with a fixed number of nodes and links, we start with a duct-layer topology the same as the link-layer topology, and then remove some ducts while keeping the link layer topology unchanged, but having the corresponding links go through multiple ducts. Note that when doing this, the network always satisfies sufficient condition 2 of Section III-A, i.e., for each edge $(i, j)$ in the duct-layer topology, there is a corresponding edge $(i, j)$ in the link-layer topology. The results are shown in Table IV. We observe that the more ducts are removed, the more wavelength links are required to route the traffic. When the duct-layer topology is the same as the link-layer topology, routing on the duct layer is equivalent to routing on the link layer. More wavelength links are required to guarantee the network's survivability from single-duct failures than that is required to guarantee the network's survivability from single-link failures. With this heuristic, the larger the difference between the two layered topologies, the larger is the difference in the number of wavelength links required. This fact reflects a tradeoff between resilience and network resources.

\section{CONCLUSION AND Future RESEARCH}

We investigated different ILP formulations and a heuristic for solving the RWA problem in a WDM mesh network when duct-layer constraints are considered and different path-protection algorithms are employed. The following summarizes the contributions of this paper:

- an ILP formulation for combined RWA with dedicated-path protection under duct-layer constraints;

- an ILP formulation for combined RWA with shared-path protection under duct-layer constraints;

- an ILP formulation for routing with duct-disjoint paths;

- two ILP formulations for wavelength assignment with shared-path protection under duct-layer constraints;
- a heuristic for RWA with shared-path protection under duct-layer constraints, which comprises three steps: a routing heuristic, a wavelength-assignment heuristic, and an optimization procedure.

We have shown using numerical examples that, for dedicatedpath protection, the total number of wavelength links is determined only by the routing algorithm, not wavelength assignment. We provide a routing heuristic whose performance is very close to the performance of the routing ILP (ILP3).

With shared-path protection, the combined RWA formulation (ILP2) is too complex to solve with limited time and computing resources. A divide-and-conquer method is employed to partition the problem into two subproblems: routing and wavelength assignment. The routing subproblem can easily be solved by the routing heuristic or ILP3. The wavelength-assignment subproblem can be solved by ILP4, ILP5, or the heuristic from Section III. ILP5 can be solved much faster than ILP4, provided that the program is fed with a set of duct-disjoint routes between each source-destination pair where there is a traffic demand. Our heuristic performs very close to ILP4 and ILP5.

This paper focused on static traffic only. With dynamic traffic, when a pair of duct-disjoint routes and a pair of wavelengths have to be determined for a given connection demand, the heuristics can be employed to solve the problem. However, with existing lightpaths in the network, some wavelengths on some links might be occupied by other connections. It is important to decide which ducts should be considered before the routing heuristic can be applied. There are at least two options.

- Consider all the ducts. This may lead to the result that there is no common wavelengths on one of the two paths.

- Construct a duct-layer topology for each wavelength. This will find a solution when a pair of duct-disjoint paths can be assigned the same wavelength. However, this method will not be able to find a solution if the primary path and the backup path have to be on different wavelengths.

\section{REFERENCES}

[1] S. Ramamurthy and B. Mukherjee, "Survivable WDM mesh networks_Part I: Protection," in Proc. IEEE INFOCOM, Mar. 1999, pp. 744-751.

[2] R. Iraschko, M. MacGregor, and W. Grover, "Optimal capacity placement for path restoration in STM or ATM mesh-survivable network," IEEE J. Select. Areas Commun., vol. 16, pp. 325-336, Sept. 1998.

[3] H. Zang and B. Mukherjee, "Connection management for survivable wavelength-routed WDM mesh networks," Opt. Netw. Mag., vol. 2, pp. 17-28, Sept. 2001.

[4] O. Gerstel, "Opportunities for optical protection and restoration," in Proc. Optical Fiber Communications Conf., 1998, pp. 269-270.

[5] G. Mohan, C. S. R. Murthy, and A. K. Somani, "Efficient algorithms for routing dependable connections in WDM optical networks," IEEE/ACM Trans. Networking, vol. 9, pp. 553-566, Oct. 2001.

[6] S. Ramamurthy and B. Mukherjee, "Survivable WDM mesh networks-Part II: Restoration," in Proc. IEEE Integrated Circuits Conf., June 1999, pp. 2023-2030.

[7] J. Strand, A. Chiu, and R. Tkach, "Issues for routing in the optical layer," IEEE Commun. Mag., vol. 39, pp. 81-87, Feb. 2001.

[8] P. Sebos, J. Yates, G. Hjalmtysson, and A. Greenberg, "Auto-discovery of shared risk link groups," in Proc. Optical Fiber Communications Conf., Mar. 2001, p. WDD3.

[9] P. Sebos, J. Yates, A. Greenberg, and D. Rubenstein, "Effectiveness of shared risk link group auto-discovery in optical networks," in Proc. Optical Fiber Communications Conf., Mar. 2002, p. ThO5.

[10] H. Zang, J. P. Jue, and B. Mukherjee, "A review of routing and wavelength assignment approaches for wavelength-routed optical WDM networks," Opt. Netw. Mag., vol. 1, pp. 47-63, Jan. 2000. 
[11] L. Sahasrabuddhe and B. Mukherjee, "Fault tolerance in IP-over-WDM networking: WDM protection versus IP restoration," IEEE J. Select. Areas Commun., vol. 20, pp. 21-33, Jan. 2002.

[12] B. T. Doshi, S. Dravida, P. Harshavardhana, O. Hauser, and Y. Wang, "Optical network design and restoration," Bell Labs Tech. J., vol. 4, pp. 58-84, Jan.-Mar. 1999.

[13] M. Sridharan, A. K. Somani, and M. V. Salapaka, "Approaches for capacity and revenue optimization in survivable WDM networks," J. High Speed Networks, vol. 10, no. 2, pp. 109-125, 2001.

[14] A. F. Fumagalli, I. Cerutti, F. B. Masetti, R. Jagannathan, and S. Alagar, "Survivable networks based on optimal routing and WDM self-healing rings," in Proc. IEEE INFOCOM, vol. 2, Mar. 1999, pp. 726-733.

[15] G. Sahin and M. Azizoglu, "Optical layer survivability: Single serviceclass case," in Proc. SPIE Opticomm, vol. 4233, Richardson, TX, Oct. 2000, pp. 267-278.

[16] S. Sengupta and R. Ramamurthy, "From network design to dynamic provisioning and restoration in optical cross-connect mesh networks: An architectural and algorithmic overview," IEEE Network, vol. 15, pp. 46-54, Jul./Aug. 2001.

[17] G. Ellinas, E. Bouillet, R. Ramamurthy, J. Labourdette, S. Chaudhuri, and K. Bala, "Routing and restoration architectures in mesh optical networks," SPIE Opt. Networks Mag., vol. 4, pp. 91-106, Jan./Feb. 2003.

[18] O. Gerstel and R. Ramaswami, "Optical layer survivability-an implementation perspective," IEEE J. Select. Areas Commun., vol. 18, pp. $1885-1899$, Oct. 2000

[19] P.-H. Ho and H. T. Mouftah, "A novel routing protocol for WDM mesh networks," in Proc. Optical Fiber Communications Conf., Mar. 2002, p. TuG4.

[20] D. Dunn, W. Grover, and M. MacGregor, "Comparison of $k$-shortest paths and maximum flow routing for network facility restoration," IEEE J. Select. Areas Commun., vol. 12, pp. 88-99, Jan. 1994

[21] M. R. Garey and D. S. Johnson, Computers and Intractability: A Guide to the Theory of NP-Completeness. New York: Freeman, 1979.

[22] J. W. Suurballe and R. E. Tarjan, "A quick method for finding shortest pairs of disjoint paths," Networks, vol. 14, pp. 325-336, 1984.

[23] D. E. Knuth, J. H. Morris, and V. R. Pratt, "Fast pattern matching in strings," SIAM J. Comput., vol. 6, no. 1, pp. 323-350, 1977.

[24] CPLEX. ILOG, Mountain View, CA. [Online]. Available: http://www.ilog.com/products/cplex

[25] S. Datta, S. Sengupta, S. Biswas, and S. Datta, "Efficient channel reservation for backup paths in optical mesh networks," in Proc. IEEE Globecom, 2001, pp. 2104-2108.

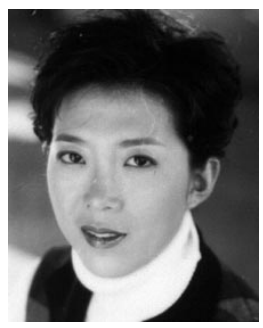

Hui Zang (S'97-M'02) received the B.S. degree in computer science from Tsinghua University, Beijing, China, in 1997, and the M.S. and Ph.D. degrees in computer science from the University of California, Davis, in 1998 and 2001, respectively.

In 2000, she joined Sprint Advanced Technology Laboratories, Burlingame, CA, where she is a Principal Applied R\&D Engineer. She was one of the Guest Editors of IEEE Network special issue on "Traffic Engineering in Optical Networks." She is the author of the book WDM Mesh Networks-Management and Survivability, (Norwell, MA: Kluwer, 2002). She has published about 30 conference papers and journal articles and currently has three U.S. patents pending in the field of optical networking. Her research interests include WDM optical networks, IP over WDM networks and quality of service.

Dr. Zang serves or has served as a Technical Committee member of IPoW'00, IPoW'01, APOC'02, DRCN'03, OptiComm'03, and Globecom'03 Optical Networking and Systems Symposium. She also helped organize OptiComm'02 as a Panel Co-chair.

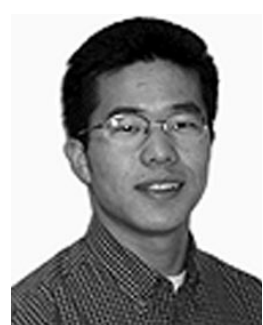

traffic grooming.

Canhui (Sam) Ou (S'02) received the B.S. degree from Beijing University, Beijing, China, in 2000 and the M.S. degree from the University of California, Davis, in 2001. He is currently working toward the $\mathrm{Ph} . \mathrm{D}$. degree in the computer science at the University of California, Davis.

He was an Intern with Sprint Advanced Technology Laboratories, Burlingame, CA, during the summer of 2001 and 2002. His research interests include design and analysis of wavelength-routed WDM networks with focus on survivability and

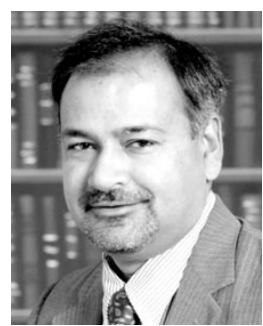

Biswanath Mukherjee (S'82-M'87) received the B.Tech. (Hons) degree from the Indian Institute of Technology, Kharagpur, India, in 1980 and the Ph.D. degree from the University of Washington, Seattle, in 1987.

In 1987, he joined the University of California, Davis, where he has been a Professor of computer science since 1995. He served as Chairman of the Department of Computer Science from 1997 to 2000. He is a Member of the Board of Directors of IPLocks Inc., a Silicon Valley startup company. He has consulted for and served on the Technical Advisory Board of a number of startup companies in optical networking. He has served on the editorial boards of the ACM/Baltzer Wireless Information Networks (WINET), the Journal of High-Speed Networks, Photonic Network Communications, and Optical Network Magazine. He is the author of the textbook Optical Communication Networks (New York: McGraw-Hill, 1997), a book which received the Association of American Publishers, Inc. 1997 Honorable Mention in Computer Science. His research interests include lightwave networks, network security, and wireless networks.

Dr. Mukherjee is a corecipient of paper awards presented at the 1991 and 1994 National Computer Security Conferences. He has served on the editorial boards of the IEEE/ACM TRANSACTIONS ON NETWORKING and IEEE Network. He has also served as Editor-at-Large for optical networking and communications for the IEEE Communications Society, and as the Technical Program Chair of the IEEE INFOCOM 1996 conference. While at the University of Washington, he held a GTE Teaching Fellowship and a General Electric Foundation Fellowship. 